\title{
Síntese e avaliação biológica de chalconas e derivados in vitro contra célula AGP01
}

\author{
Synthesis and biological evaluation of chalcones and derivatives in vitro against AGP01 cell \\ Síntesis y evaluación biológica de chalconas y derivados in vitro frente a células AGP01
}

Recebido: 19/11/2021 | Revisado: 27/11/2021 | Aceito: 04/12/2021 | Publicado: 13/12/2021

\author{
Maricelia Lopes dos Anjos \\ ORCID: https://orcid.org/0000-0001-6837-0276 \\ Universidade Federal do Pará, Brasil \\ E-mail: mariceliadosanjos@yahoo.com.br \\ Herald Souza dos Reis \\ ORCID: https://orcid.org/0000-0001-6513-3937 \\ Universidade Federal do Pará, Brasil \\ E-mail: herald.reis@live.com \\ Heriberto Rodrigues Bitencourt \\ ORCID: https://orcid.org/0000-0002-0003-2876 \\ Universidade Federal do Pará, Brasil \\ E-mail: heriberto.ufpa@gmail.com \\ Natasha Costa da Rocha Galucio \\ ORCID: https://orcid.org/0000-0003-4923-1478 \\ Universidade Federal do Pará, Brasil \\ E-mail: natashagalucio@gmail.com \\ André Salim Khayat \\ ORCID: https://orcid.org/0000-0002-3451-6369 \\ Universidade Federal do Pará, Brasil \\ E-mail: khayatas@gmail.com \\ Antônio Pedro da Silva Souza Filho \\ ORCID: https://orcid.org/0000-0001-9213-2139 \\ Embrapa Amazônia Oriental, Brasil \\ E-mail: antonio-pedro.filho@embrapa.br \\ Marta Chagas Monteiro \\ ORCID: https://orcid.org/0000-0002-3328-5650 \\ Universidade Federal do Pará, Brasil \\ E-mail: martachagas@ufpa.br
}

\section{Resumo}

O interesse pela obtenção de chalconas se deve as inúmeras atividades farmacológicas descritas na literatura para esses compostos que são intermediários da biossíntese de flavonoides. Dessa forma, a síntese e caracterização de várias chalconas e derivados se tornam importantes para o desenvolvimento de compostos com atividade antiproliferativa. Neste trabalho foram sintetizadas dez substâncias, sendo sete chalconas e três naftoflavanonas, obtidas pela reação de condensação aldólica de Claisen Schmidt entre acetofenonas e aldeídos, em condições de temperatura ambiente em meio básico. Os rendimentos variaram entre $65,35 \%$ e 97,45\%. Sendo caracterizadas e confirmadas por meio de técnicas espectroscópicas de RMN de ${ }^{1} \mathrm{H}$ e ${ }^{13} \mathrm{C}$ em comparação com os dados da literatura. Todos os produtos sintetizados foram submetidos ao ensaio de citotoxicidade do brometo de 3- (4,5-dimetiltiazol-2-il) -2,5-difeniltetrazólio (MTT), para a avaliação da atividade antiproliferativa in vitro, em linhagem tumoral gástrica (AGP01), com comparação à linhagem celular normal de pulmão (MRC-5). As substancias mais ativas, foram as chalconas 2, 6, 3, 4 e 1 que apresentaram maior redução na viabilidade celular de AGP01, sendo maior que $90 \%$.

Palavras-chave: Chalcona; Flavanonas; Antiproliferativa.

\begin{abstract}
The interest in obtaining chalcones is due to the numerous pharmacological activities described in the literature for these compounds that are intermediates in the biosynthesis of flavonoids. Thus, the synthesis and characterization of several chalcones and derivatives become important for the development of compounds with antiproliferative activity. In this work, ten substances were synthesized, seven chalcones and three naphthoflavanones, obtained by the Claisen Schmidt aldol condensation reaction between acetophenones and aldehydes, at room temperature conditions in a basic medium. Yields ranged between $65.35 \%$ and $97.45 \%$. Being characterized and confirmed through ${ }^{1} \mathrm{H}$ and ${ }^{13} \mathrm{C}$ NMR spectroscopic techniques in comparison with literature data. All synthesized products were submitted to the cytotoxicity assay of 3-(4,5-dimethylthiazol-2-yl)-2,5-diphenyltetrazolium (MTT) bromide, to evaluate the antiproliferative activity in vitro, in gastric tumor lineage (AGP01), compared to the normal lung cell line (MRC-5). The most active substances were chalcones 2, 6, 3, 4 and 1, which showed the greatest reduction in cell viability of AGP01, being greater than $90 \%$.
\end{abstract}

Keywords: Chalcone; Flavanones; Antiproliferative. 


\section{Resumen}

El interés en la obtención de calconas se debe a las numerosas actividades farmacológicas descritas en la literatura para estos compuestos que son intermediarios en la biosíntesis de flavonoides. De esta forma, la síntesis y caracterización de varias calconas y derivados adquiere importancia para el desarrollo de compuestos con actividad antiproliferativa. En este trabajo se sintetizaron diez sustancias, siete chalconas y tres naftoflavanonas, obtenidas mediante la reacción de condensación aldólica de Claisen Schmidt entre acetofenonas y aldehídos, a temperatura ambiente en un medio básico. Los rendimientos oscilaron entre el 65,35\% y el 97,45\%. Estar caracterizado y confirmado mediante técnicas espectroscópicas de $\mathrm{RMN}{ }^{1} \mathrm{H}$ y ${ }^{13} \mathrm{C}$ en comparación con los datos de la literatura. Todos los productos sintetizados se sometieron al ensayo de citotoxicidad de bromuro de 3- (4,5-dimetiltiazol-2-il) -2,5difeniltetrazolio (MTT), para evaluar la actividad antiproliferativa in vitro, en linaje tumoral gástrico (AGP01), en comparación con la línea de células pulmonares normales (MRC-5). Las sustancias más activas fueron las chalconas 2, 6, 3, 4 y 1 que mostraron la mayor reducción en la viabilidad celular de AGP01, siendo superior al 90\%.

Palabras clave: Chalcona; Flavanonas; Antiproliferativo.

\section{Introduçãa}

O câncer é uma das principais causas de morte no mundo, sendo considerado um dos principais problema de saúde pública. Genericamente o câncer é definido como um grupo de doenças que podem afetar qualquer parte do corpo. O câncer gástrico (CG) é o quinto mais diagnosticado (1,03 milhão) de casos e a terceira principal causa de morte por câncer no mundo, tendo 783 mil mortes em 2018, de acordo com dados estabelecidos pelo IARC, para 36 tipos de câncer em 185 países (WHO, 2018; Bray et al., 2018; Ferlay et al., 2019; Plummer et al., 2016). Apesar do enorme progresso no tratamento para essa doença, a cirurgia continua sendo o principal tratamento para o CG (Orditura et al., 2014; Marrelli et al., 2015).

Em razão de estratégias de tratamento ineficazes e a resistência de tumores à quimioterapia e radioterapia e pela incapacidade de medicamentos quimioterápicos não diferenciarem as células saudáveis das células cancerígenas, proporcionando resultado clínico indesejado, o câncer continua progredindo significativamente em todo o mundo (Alard et al., 2020; Fiorica et al., 2018; Yadav et al., 2017). Dessa forma, faz-se necessário urgentemente uma abordagem promissora para o avanço nas pesquisas de novos agentes antiproliferativos mais efetivos, seletivos e com baixa toxicidade em células saudáveis.

Dentre os compostos com ações em diversas patologias, destacam-se as chalconas que pertencem a classe dos flavonoides (Sandhar et al., 2011; Isoda et al., 2014; Wang, Li \& Bi, 2018). As chalconas são definidas como flavonoides de cadeia aberta, onde possuem duas subunidades benzênicas (anéis A e B) espaçadas por uma cetona $\alpha, \beta$-insaturada, compondo um esqueleto C6-C3-C6, sendo 1,3-difenil-2-propen-1-ona a sua estrutura básica (Figura 1) (Sharma et al., 2016; Das \& Manna, 2016; Ahmad et al., 2016).

Figura 1- Estrutura química básica de chalcona, benzoflavanona e naftoflavanona.

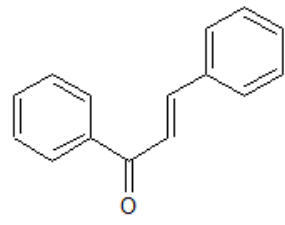

Chalcona

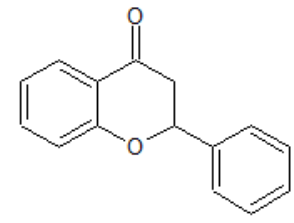

Benzoflavanona<smiles>O=C1CC(c2ccccc2)Oc2ccc3ccccc3c21</smiles>

Naftoflavano na

Fonte: Autores.

As chalconas vem sendo descritas na literatura por possuírem grande potencial farmacológico, incluindo atividade antioxidante (Ahmad et al., 2016 ), antiviral (Fu et al., 2020), antinociceptivo (Ismail et al., 2016), antimalárica (Suwito et al., 2014; Tomar et al., 2010), leishimanicida (De Mello et al., 2014), quimiopreventivo e quimioterápico (Orlikova et al., 2011), antimicrobiana (Ventura et al., 2015), anti-inflamatória (Wu et al., 2011 ), nematicida (Attar et al., 2011), antifúngica (Zhang et al., 2011), citotóxica (Ahmad et al., 2016), antiproliferativa contra células cancerígenas de MCF-7 (câncer de mama) (Yadav 
et al., 2017; Santos et al., 2019), A549 (câncer de pulmão) (Yadav et al., 2017), PC-3 (próstata) (Marquina et al., 2019), HepG2 (câncer de fígado) (Yadav et al., 2017), HT29 (cólon) (Kotha et al., 2017), dentre outras atividades.

Tendo em vista a importância biológica desses compostos para a descoberta de novos fármacos e a busca por agentes anticancerígenos a partir de estruturas baseadas nas chalconas, relata-se aqui a síntese e a avaliação do potencial citotóxico in vitro de chalconas e derivados, naftoflavanonas, contra a linhagem AGP01 (adenocarcinoma gástrico) com comparação à linhagem MRC-5 (célula normal de pulmão).

\section{Material e Métodos}

\subsection{Procedimentos gerais}

Os espectros de $\mathrm{RMN}{ }^{1} \mathrm{H}$ e ${ }^{13} \mathrm{C}$ foram registrados em um espectrômetro Ascend 400 (Bruker) operando a $400 \mathrm{MHz}$ e Varian Mercury-300 MHz, usando $\mathrm{CDCl}_{3}$ como solvente. Os deslocamentos químicos $(\delta)$ são dados em valores de ppm a partir do TMS e as constantes de acoplamento (J) em Hertz (Hz).

\subsection{Células}

As células, Adenocarcinoma Gástrico (AGP01) e Fibroblasto de pulmão humano (MRC5), foram obtidas de uma coleção do Núcleo de pesquisa em oncologia - NPO na Universidade federal do Pará, Brasil.

\subsection{Substâncias}

\subsubsection{Procedimento de obtenção das substâncias}

Foram sintetizadas dez substâncias, sendo sete chalconas e três flavanonas, suas estruturas básicas são mostradas na Tabela1. As sínteses foram realizadas no laboratório da Faculdade de Química da Universidade Federal do Pará, Belém, Brasil. O procedimento geral da obtenção das substâncias foi, adição de solvente (15 mL, EtOH ou MeOH), cetona ( 10 mmol), catalisador (15 mL de solução de $\mathrm{NaOH}$ a 10\%) e o aldeído ( 10mmol com excesso de 10\%) foram adicionados sequencialmente em banho de gelo. A mistura reacional foi mantida sob agitação magnética à temperatura ambiente durante 3 horas. Em seguida, foi resfriado e deixado no freezer por 24 horas. Após esse período, foi realizado uma acidificação com ácido acético (25\%), no caso dos compostos fenólicos e então foi realizada a extração com acetato de etila ou clorofórmio, secagem e evaporação do solvente. O produto obtido foi recristalizado em acetato de etila (Bitencourt et al., 2020a e 2020b).

\subsubsection{Dados espectroscópicos das substâncias sintetizadas}

- (2E) -1,3-difenilprop-2-en-1-ona (1): cristal amarelo claro, P.f. experimental 54-55 ${ }^{\circ}$, rendimento $91,23 \%$ e fórmula molecular $\mathrm{C}_{16} \mathrm{H}_{14} \mathrm{O}$.

$\mathbf{R M N}^{1} \mathbf{H}\left(400 \mathrm{MHz} ; \mathrm{CDCl}_{3}\right) \delta_{(\mathrm{ppm})}:$ 7,84 (d, 1H, J=15,6Hz, H-7), 7,56 (d, 1H, J=15,6Hz, H-8), 7,43-8,06 (m, $\left.10 \mathrm{H}, \mathrm{H}-\mathrm{Ar}\right)$.

$\mathbf{R M N}^{13} \mathbf{C}\left(100 \mathrm{MHz} ; \mathrm{CDCl}_{3}\right) \delta_{(\mathrm{ppm})}: 138,2(\mathrm{C} 1), 128,4$ (C2), 128,9 (C3), 128,6 (C4), 128,5 (C5), 128,4 (C6), 144,9 (C7), 122,1 (C8), 190,6 (C9), 130,5 (C1’'), 134,9 (C2`), 77,4 (C3`), 77,1 (C4`), 76,8 (C5'), 132,8 (C-6’).

- (2E) -1-fenil-3-(2-metóxi-fenil) -prop-2-en-1-ona (2): cristal amarelo claro, P.f. experimental 58-59 C, rendimento 67,35\% e fórmula molecular $\mathrm{C}_{16} \mathrm{H}_{14} \mathrm{O}_{2}$.

$\mathbf{R M N}^{1} \mathbf{H}\left(400 \mathrm{MHz} ; \mathrm{CDCl}_{3}\right) \delta_{(\mathrm{ppm})}:$ 8, 15 (d, 1H, J=16Hz, H-7), 7,65 (d, 1H, J=16Hz, H-8), 6,95-8,05 (m, 9H, H-Ar), 3,99 (s,3H, $\left.\mathrm{OCH}_{3}\right)$.

$\mathbf{R M N}^{13} \mathbf{C}\left(100 \mathrm{MHz} ; \mathrm{CDCl}_{3}\right) \delta_{(\mathrm{ppm})}:$ 132,5 (C1), 120,7 (C2), 111,2 (C3), 123,9 (C4), 138,5 (C5), 158,8 (C6), 140,4 (C7), 122,8

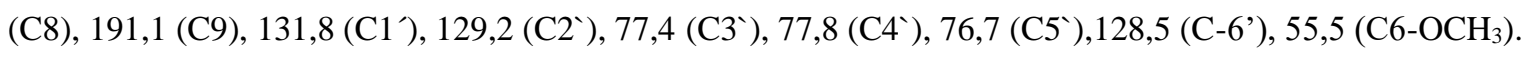


- (2E) -1-fenil-3-(3-metóxi-fenil) -prop-2-en-1-ona (3): cristal castanho claro, P.f. experimental 58-59² , rendimento 65,35\% e fórmula molecular $\mathrm{C}_{16} \mathrm{H}_{14} \mathrm{O}_{2}$.

$\mathbf{R M N}^{1} \mathbf{H}\left(400 \mathrm{MHz} ; \mathrm{CDCl}_{3}\right) \delta_{(\mathrm{ppm})}:$ 7,80 (d, 1H, J=16Hz, H-7), 7,36 (d, 1H, J=16Hz, H-8), 6,98-8,05 (m, 9H, H-Ar), 3,88 (s,3H, $\left.\mathrm{OCH}_{3}\right)$.

$\mathbf{R M N}^{13} \mathbf{C}\left(100 \mathrm{MHz} ; \mathrm{CDCl}_{3}\right) \delta_{(\mathrm{ppm})}:$ 136,2 (C1), 121,1 (C2), 128,5 (C3), 116,3 (C4),159,9 (C5), 113,4 (C6), 144,8 (C7), 122,4 (C8), 190,6 (C9), 132,8 (C1`), 129,9 (C2`), 77,3 (C3`), 77,0 (C4`), 76,7 (C5`), 128,6 (C-6’), 55,3 (C5-OCH $\left.{ }_{3}\right)$.

- (2E) -1-fenil-3-(4-metóxi-fenil) -prop-2-en-1-ona (4): cristal amarelo claro, P.f. experimental 71-72 C, rendimento 90,30\% e fórmula molecular $\mathrm{C}_{16} \mathrm{H}_{14} \mathrm{O}_{2}$.

$\mathbf{R M N}^{1} \mathbf{H}\left(400 \mathrm{MHz} ; \mathrm{CDCl}_{3}\right) \delta_{(\mathrm{ppm})}:$ 7,81 (d, 1H, J=15,6Hz, H-7), 7,44 (d, 1H, J=15,6Hz, H-8), 7,62-7,64 (d, 2H, H-2 e H-6), 6,95-6,97 (d,2H, H-3 e H-5), 7,50-8,04 (m, 5H, H-Ar), 3,88 (s, 3H, $\left.\mathrm{OCH}_{3}\right)$.

$\mathbf{R M N}^{13} \mathbf{C}\left(100 \mathrm{MHz} ; \mathrm{CDCl}_{3}\right) \delta_{(\mathrm{ppm})}:$ 138,5 (C1), 128,4 (C2), 114,4 (C3), 161,7 (C4), 159,9 (C5), 113,4 (C6), 144,8 (C7), 119,8 (C8), 190,6 (C9), 132,5 (C1`), 130,2 (C2`), 77,3 (C3`), 77,0 (C4`), 76,7 (C5`), 128,5 (C-6’), 55,4 (C4-OCH $\left.{ }_{3}\right)$.

- (2E) -1-(4-metóxi-fenil) -3-fenil-prop-2-en-1-ona (5): cristal amarelo claro, P.f. experimental 105-106 ${ }^{\circ} \mathrm{C}$, rendimento $87,93 \%$ e fórmula molecular $\mathrm{C}_{16} \mathrm{H}_{14} \mathrm{O}_{2}$.

$\mathbf{R M N}^{1} \mathbf{H}\left(400 \mathrm{MHz} ; \mathrm{CDCl}_{3}\right) \delta_{(\mathrm{ppm})}:$ 7,82 (d, 1H, J=15,6Hz, H-7), 7,57 (d, 1H, J=15,6Hz, H-8), 8,05-8,08 (d, 2H, H-3' e H-5'), 7,00-7,02 (d,2H, H-2' e H-6'), 7,43-7,68 (m, 5H, H-Ar), 3,91 (s, 3H, $\left.\mathrm{OCH}_{3}\right)$.

$\mathbf{R M N}^{13} \mathbf{C}\left(100 \mathrm{MHz} ; \mathrm{CDCl}_{3}\right) \delta_{(\mathrm{ppm})}: 131,1(\mathrm{C} 1), 113,8$ (C2), 77,3 (C3), 77,0 (C4), 76,7 (C5), 113,8 (C6), 143,9 (C7), 121,9 (C8), 188,7 (C9), 135,1 (C1’), 128,9 (C2`), 130,8 (C3`), 163,4 (C4`), 130,3 (C5 '), 128,3 (C-6’), 55,5 (C4’-OCH $)$.

-(2E) -1-(4-metóxi-fenil) -3-(3,4,5-Tri-metóxi-fenil) -prop-2-en-1-ona (6): cristal amarelo intenso, P.f. experimental 129$130^{\circ} \mathrm{C}$, rendimento $97,45 \%$ e fórmula molecular $\mathrm{C}_{19} \mathrm{H}_{20} \mathrm{O}_{5}$.

$\mathbf{R M N}^{1} \mathbf{H}\left(400 \mathrm{MHz} ; \mathrm{CDCl}_{3}\right) \delta_{(\mathrm{ppm})}: 7,73$ (d, 1H, J=15,6Hz, H-7), 7,43 (d, 1H, J=15,6Hz, H-8), 8,04-8,07 (d, 2H, H-3' e H-5'),

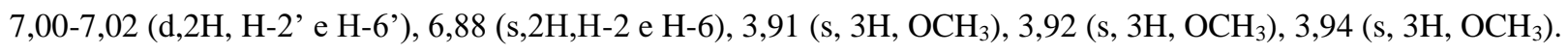

$\mathbf{R M N}^{13} \mathbf{C}\left(100 \mathrm{MHz} ; \mathrm{CDCl}_{3}\right) \delta_{(\mathrm{ppm})}: 77.3$ (C1), 105,6 (C2), 153,4 (C3), 140,3 (C4), 130,8 (C5), 105,6 (C6), 144,1 (C7), 121,2 (C8), 188,7 (C9), 113,8 (C1’), 113,8 (C2`), 131,1 (C3`), 163,4 (C4`), 130,5 (C5 ), 113,8 (C-6’), 61,0 (C4’-OCH3), 56,2 (C3$\left.\mathrm{OCH}_{3}\right), 55,5\left(\mathrm{C} 4-\mathrm{OCH}_{3}\right), 55,5\left(\mathrm{C} 5-\mathrm{OCH}_{3}\right)$.

-(2E) -1-(4-hidróxi-fenil) -3-(4-metóxi-fenil) -prop-2-en-1-ona (7): cristal amarelo claro, P.f. experimental 115-129 C e rendimento $74,36 \%$ e fórmula molecular $\mathrm{C}_{16} \mathrm{H}_{14} \mathrm{O}_{3}$.

$\mathbf{R M N}^{1} \mathbf{H}\left(400 \mathrm{MHz} ; \mathrm{CDCl}_{3}\right) \delta_{(\mathrm{ppm})}: 7,80$ (d, 1H, J=15,6Hz, H-7), 7,44 (d, 1H, J=15,6Hz, H-8), 7,99-8,02 (d, 2H, H-2 e H-6), 7,72-7,61 (d,2H, H-3' e H-5'), 6,94-6,97 (H-3-H-5), 7,58-7,62 (H-2'-H-6'), 3,87 (s, 3H, OCH ). $^{\prime}$

$\mathbf{R M N}^{13} \mathbf{C}\left(100 \mathrm{MHz} ; \mathrm{CDCl}_{3}\right) \delta_{(\mathrm{ppm})}: 143,1$ (C1), 127,7 (C2), 114,4 (C3), 160,3 (C4), 114,4 (C5), 127,5 (C6), 144,2 (C7), 123,3 (C8), 189,2 (C9), 161,6 (C1’), 131,1 (C2`), 130,9 (C3`), 161,6 (C4`), 130,1 (C5'),131,1 (C-6’), 55,4 (C4-OCH $\left.{ }^{\prime}\right)$.

-Nafto [2,1] - piran-1-ona,2,3-di-hidro-3-(2-metóxi-fenil) (8): cristal amarelo claro, P.f. experimental $141-143^{\circ} \mathrm{C}$, rendimento $85,44 \%$ e fórmula molecular $\mathrm{C}_{20} \mathrm{H}_{16} \mathrm{O}_{3}$.

$\mathbf{R M N}^{1} \mathbf{H}\left(400 \mathrm{MHz} ; \mathrm{CDCl}_{3}\right.$ ) $\delta(\mathrm{ppm}): 6,02$ (dd, 1H, J=8,4Hz, H-7), 3,09-3,10 (dd, 2H, J=9,2Hz e J=1,6Hz, H8a e H8b), 6,967,12 (m, 4H,H-Ar), 9,54 (ddd, 2H, J=8,8, H-8'), 7,36 (dd, 2H, J=9Hz, H-3'), 7,98 (dd, 2H, J=9Hz, H-4'), 7,80 (ddd, 2H, $\mathrm{J}=1,2 \mathrm{~Hz}, \mathrm{~J}=0,6 \mathrm{~Hz}, \mathrm{H}-5^{\prime}$ '), 7,66 (ddd, 1H, J=0,9Hz, H-6'), 7,69 (ddd, 2H, J=3,3Hz, J=1,5Hz, H-7'), 3,88 (s, 3H, OCH3). 
RMN'13C (100MHz; $\mathrm{CDCl}_{3}$ ) $\delta(\mathrm{ppm}):$ 127,2 (C1), 155,9 (C2), 110,5 (C3), 126,4 (C4), 129,4 (C5), 120,8 (C6), 77,3 (C7), 44,8 (C8), 193,7 (C9), 55,4 (C2-OCH3),112,6 (C1'), 155,9 (C2 '), 118,9 (C3`), 137,3 (C4`), 129,2 (C4a’) 128,3 (C5 ) 124,8 (C-6`), 129,6 (C7'), 125,9 (C8'), 131,6 (C8a').

- Nafto [2,1] -piran-1-ona,2,3-di-hidro-3-(3-metóxi-fenil) (9): cristal castanho claro, P.f. experimental 105-106 ${ }^{\circ} \mathrm{C}$, rendimento $93,04 \%$ e fórmula molecular $\mathrm{C}_{20} \mathrm{H}_{16} \mathrm{O}_{3}$.

$\mathbf{R M N}^{1} \mathbf{H}\left(300 \mathrm{MHz} ; \mathrm{CDCl}_{3}\right.$ ) $\delta(\mathrm{ppm}):$ 7,08-7,09 (ddd, 2H, H-2 e H-6), 6,93 (ddd, 1H, J=1,8Hz e J=0,6Hz, H-4), 7,39 (ddd, $1 \mathrm{H}, \mathrm{J}=5,4 \mathrm{~Hz}$ e J=2,4Hz, H-5), 5,57 (dd, 1H, J=3Hz, H-7), 3.20-2,99 (dd, 2H, J=13,20Hz e J=3Hz, H8a e H8b), 3,85 (s, 3H, OCH3), 7,19 (dd, 2H, J=9Hz, H-3'), 7,93 (dd, 2H, J=9Hz, H-4'), 7,77 (ddd, 2H, J=1,2Hz, J=0,6Hz, H-5'), 7,47 (ddd, 1H, $\mathrm{J}=0,9 \mathrm{~Hz}, \mathrm{H}-6$ ') $, 7,66$ (ddd, 2H, J=3,3Hz, J=1,5Hz, H-7'), 9,51 (ddd, 2H, J=8,7, H-8').

$\mathbf{R M N}^{13} \mathbf{C}\left(75 \mathrm{MHz} ; \mathrm{CDCl}_{3}\right) \delta(\mathrm{ppm}):$ 139,9 (C1), 111,8 (C2), 159,8 (C3), 113,9 (C4),129,9 (C5), 118,2 (C6), 79,3 (C7), 45,6 (C8), 192,8 (C9), 55,2 (C3-OCH3),112,4 (C1'), 163,6 (C2`), 118,7 (C3`), 137,2 (C4`), 129,1 (C4a') 128,3 (C5`),124,8 (C-6`), 129,6 (C7'), 125,8 (C8'), 131,3 (C8a').

- Nafto [2,1] -piran-1-ona,2,3-di-hidro-3-(4-metóxi-fenil) (10): cristal amarelo intenso, P.f. 118-121 ${ }^{\circ} \mathrm{C}$, rendimento 85,72\% e fórmula molecular $\mathrm{C}_{20} \mathrm{H}_{16} \mathrm{O}_{3}$.

$\mathbf{R M N}^{1} \mathbf{H}\left(400 \mathrm{MHz} ; \mathrm{CDCl}_{3}\right.$ ) $\delta: 5,59$ (dd, 1H, J=2,8Hz, H-7), 2,94-3,31 (dd, 2H, J=13,20Hz e J=2,8Hz, H8a e H8b), 8,11 (ddd, $2 \mathrm{H}, \mathrm{J}=8,4, \mathrm{H}-8^{\prime}$ ) $, 7,21$ (dd, 2H, J=9Hz, H-3'), 7,98 (dd, 2H, J=9Hz, H-4'), 7,80 (ddd, 2H, J=1,2Hz, J=0,6Hz, H-5'), 7,55 (ddd, 1H, J=0,9Hz, H-6'), 7,61 (ddd, 2H, J=3,3Hz, J=1,5Hz, H-7'), 7,38-7,49 (d,2H, H-3 e H-5), 7,90-7,93 (H-2-H-6), 3,88 (s, 3H, $\mathrm{OCH} 3)$.

$\mathbf{R M N}^{13} \mathbf{C}\left(75 \mathrm{MHz} ; \mathrm{CDCl}_{3}\right.$ ) $\delta(\mathrm{ppm}): 143,1$ (C1), 136,5 (C2), 127,7 (C3), 161,8 (C4), 127,5 (C5), 127,8 (C6), 116,0 (C7), 45,5 (C8), 194,5 (C9), 55,4 (C4-OCH3),114,5 (C1`), 125,1 (C2`), 119,4 (C3`), 137,5 (C4`), 129,2 (C4a’) 128,6 (C5`),124,7 (C-6`), 129,6 (C7'), 125,8 (C8'), 130,5 (C8a').

\subsection{Antiproliferativo}

\subsubsection{Cultura de células}

As células AGP01 foram cultivadas em meio DMEM - Dulbecco's modified Eagle's médium (Sigma) - suplementado com $10 \%$ de soro fetal bovino - SFB (Laborclin), $100 \mu \mathrm{g} / \mathrm{mL}$ de estreptomicina (Sigma) e $60 \mu \mathrm{g} / \mathrm{mL}$ de penicilina (Sigma). Esta linhagem foi cultivada em frascos de cultura T-75, armazenadas em incubadora de gás $\mathrm{CO}_{2}(\mathrm{Laboven})$, a $37^{\circ} \mathrm{C}$ em atmosfera úmida com $5 \%$ de $\mathrm{CO}_{2}$.

\subsubsection{Ensaio de viabilidade celular}

O ensaio de viabilidade celular foi realizado de acordo com a metodologia descrita por Mossman et al. (1983). Para a triagem inicial, as células de AGP01 foram cultivadas em densidade de 2 x $10^{5}$ em placas de 96 poços e incubadas com as substâncias 1-10 por $72 \mathrm{~h}$ em concentração única de $10 \mu \mathrm{g} / \mathrm{mL}$. As células de linhagem de câncer gástrico (AGP01) e de linhagem normal de pulmão (MRC5) foram incubadas com as substâncias selecionadas (1, 2, 3, 4 e 6), por 24, 48 e 72 horas nas densidades de $8 \times 10^{5}, 6 \times 10^{5}, 2 \times 10^{5}$ respectivamente em 96 poços nas concentrações de $0.125,0.25,0.5,1,2,4$ e 8 $\mu \mathrm{g} / \mathrm{mL}$ para determinar a $\mathrm{IC}_{50}$. Posteriormente as substâncias que apresentaram viabilidade celular abaixo de $50 \%$ na linhagem gástrica AGP01 foram escolhidas para os ensaios de investigação da $\mathrm{EC}_{50 \%}$ da curva dose-resposta, ou seja, a concentração do fármaco que induz metade do efeito máximo observado. 


\subsection{Análise estatística}

Os resultados foram analisados estatística utilizando ANOVA seguido do teste de Tukey para as comparações múltiplas. As análises foram realizadas no programa GraphPad Prism 6.0 ${ }^{\circledR}$. Os valores de $\mathrm{p}$ inferior ou igual a 0,05 foram considerados estatisticamente significativos.

\section{Resultados e discussão}

Sintetizou-se dez substâncias sendo sete chalconas (1-7) e três naftoflavanonas (8-10), suas estruturas químicas apresentadas na Tabela 1. Os compostos obtidos tiveram rendimentos entre 65,35\% e 97,45\% e para todas as substâncias foram realizadas análises de $\mathrm{RMN}^{1} \mathrm{H}, \mathrm{RMN}{ }^{13} \mathrm{C}$ e obtenção do Ponto de fusão.

Tabela 1 - Estrutura química das chalconas e naftoflavanonas sintetizadas.

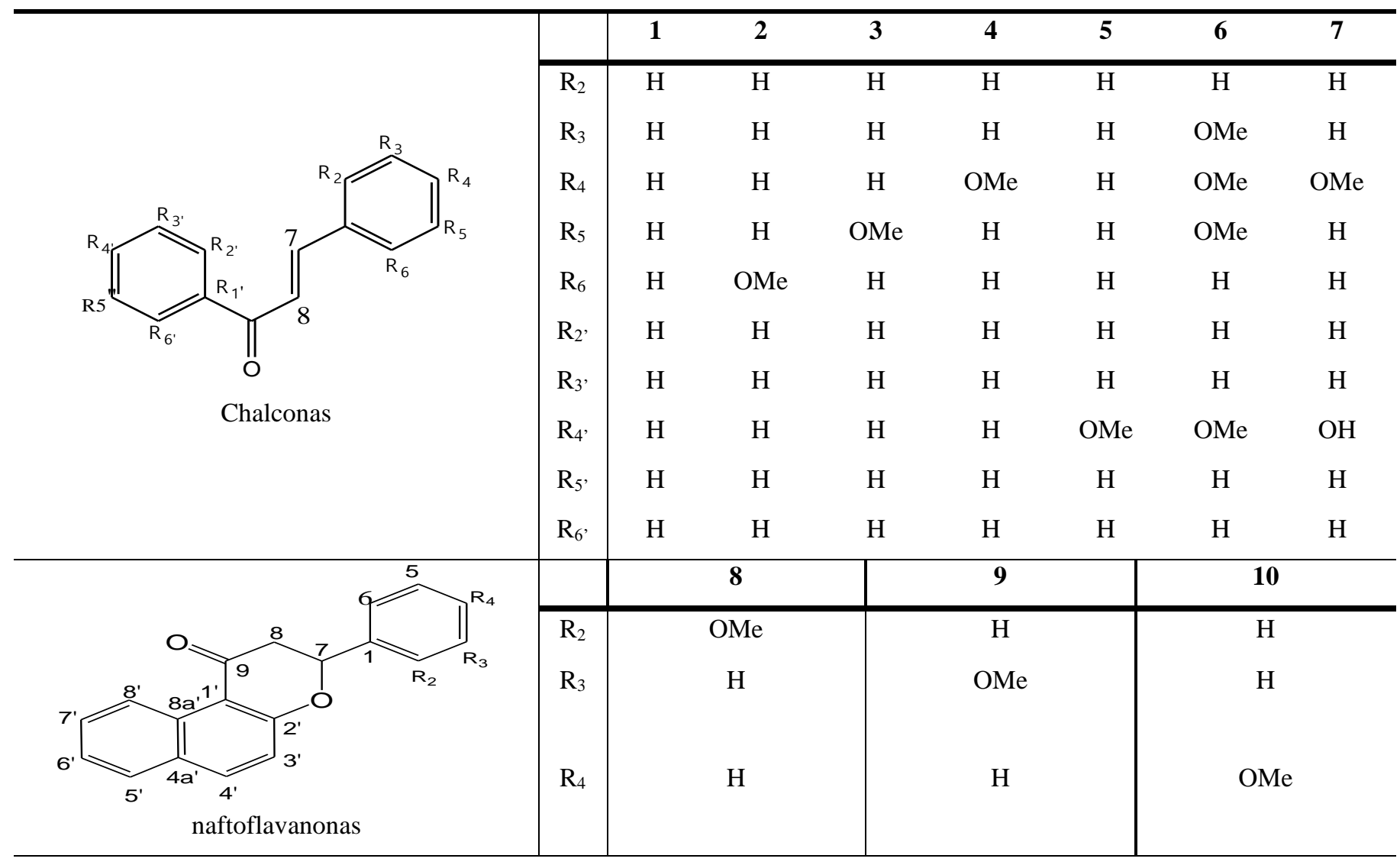

Fonte: Autores.

Para as chalconas os espectros de $\mathrm{RMN}^{1} \mathrm{H}\left(400 \mathrm{MHz} ; \mathrm{CDCl}_{3}\right)$, foram identificados os sinais relativos aos hidrogênios H-8 e H-7 como um sistema do tipo $\mathrm{AB}$, como um dupleto de constante de acoplamento de $\mathrm{J} \sim 16 \mathrm{~Hz}$ caracterizando a conformação trans das chalconas. Também, os sinais relativos aos hidrogênios aromáticos e aqueles relativos aos hidrogênios das metoxilas. No anel A das substâncias 5, 6 e 7, foi verificado o sistema AA'BB' de H-3'-H-5' e H-2'-H-6', com os sinais relativos entre 8,5-8,8 e 7,00-7,02 ppm respectivamente. Os hidrogênios H-3-H-5 e H-2-H-6 no anel B, são equivalentes para as estruturas das chalconas 4 e 7, em 6,94-6,97 e 7,62-7,64 ppm respectivamente.

As demais substancias que não apresentam substituintes para as estruturas, os sinais relativos hidrogênio aromáticos são observados como multipleto (m, 10H) em 6,95-7,68 ppm. 
No espectro de $\mathrm{RMN}^{13} \mathrm{C}\left(100 \mathrm{MHz} ; \mathrm{CDCl}_{3}\right)$ das chalconas, foram identificados os sinais relativos aos carbonos aromáticos, aos carbonos olefinícos (C7 e C8), aquele relativo ao carbono carbonílico (C9) e aos carbonos da metóxila.

Nas naftoflavanonas nos espectros de $\mathrm{RMN}^{1} \mathrm{H}\left(300 \mathrm{MHz}\right.$ e $\left.400 \mathrm{MHz} ; \mathrm{CDCl}_{3}\right)$, foram identificados os sinais relativos aos hidrogênios H-8', H-8 e H-7. Também, os sinais relativos aos hidrogênios aromáticos e aqueles relativos aos hidrogênios da metóxila $(\delta: 3,88 ; \mathrm{s} ; 3 \mathrm{H})$.

Neste estudo, visando determinar o potencial citotóxico in vitro das chalconas e derivados, foram submetidas as dez substâncias (1-10) para avaliação da viabilidade celular através do teste de MTT, sendo realizado através do teste de viabilidade celular (MTT) conforme Mosmann (1983) contra a linhagem AGP01 (adenocarcinoma gástrico). Os resultados são apresentados como porcentagem da viabilidade celular. Inicialmente realizou-se a triagem primaria e as células foram tratadas por $72 \mathrm{~h}$ em uma única concentração de $10 \mu \mathrm{g} / \mathrm{mL}$, como mostrado no Gráfico 1 , onde cinco chalconas reduziram a viabilidade celular em mais de $50 \%$, sendo essas selecionadas para estudo posterior.

Gráfico 1. Viabilidade celular da linhagem de câncer gástrico AGP01 após 72h de tratamento com as substâncias 5, 10, 8, 7, 9, $\mathbf{2}, \mathbf{1}, \mathbf{6}, \mathbf{4}$, e $\mathbf{3}$ na concentração de $10 \mu \mathrm{g} / \mathrm{mL}$.

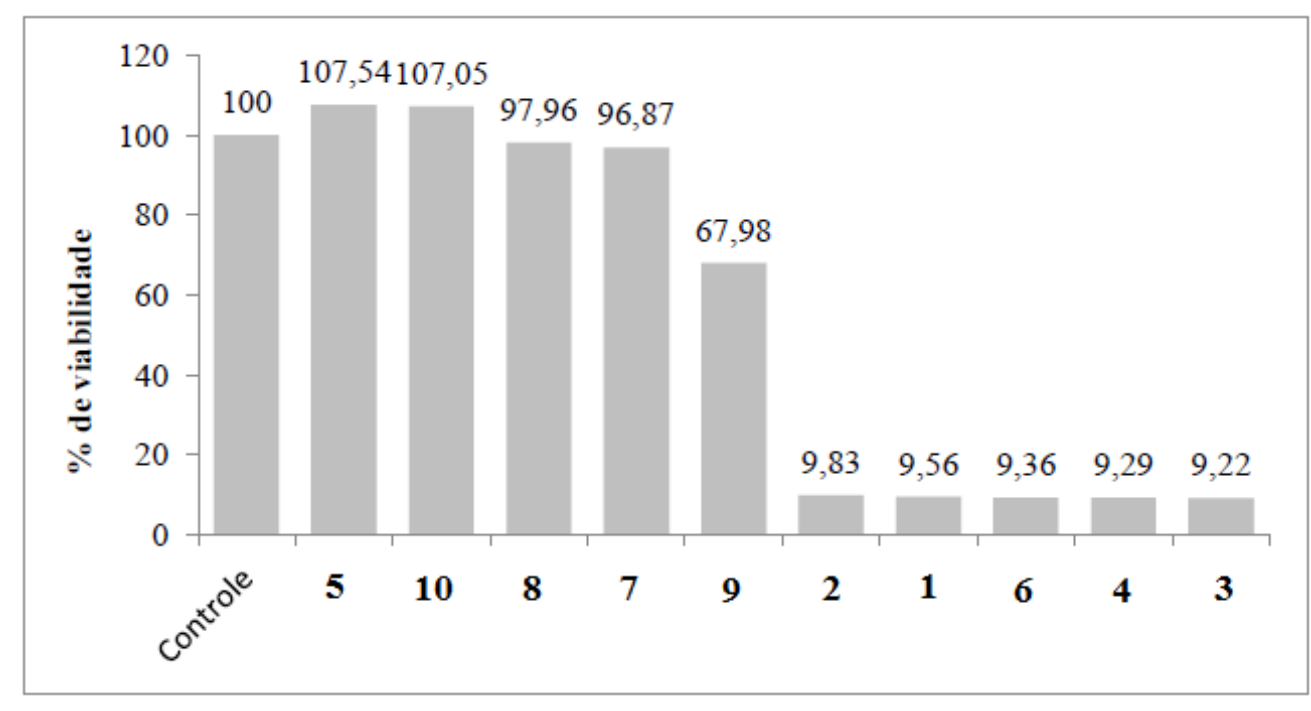

Fonte: Autores.

Entre as substâncias avaliadas, as chalconas 3, 4, 6, 1 e 2 foram as que apresentaram maior redução na viabilidade celular de AGP01 respectivamente, todas com redução maior que 90\%. Para as substâncias 5, 10, 8, 7 e 9 a viabilidade celular pouco foi diminuída em baixa concentração, sendo descartadas para estudo em linhagem normal, visto que compostos em altas concentrações não são de interesse farmacológico (Mosmann, 1983).

Os compostos com maior redução na viabilidade celular de AGP01 foram selecionados também para estudos com células normais de pulmão (MRC5) em tempos de exposição de 24, 48 e 72h. Os resultados foram tabelados e expressos na metade da concentração inibitória máxima $\left(\mathrm{IC}_{50}\right)$ como mostrado na Tabela 2 . 
Tabela 2 - Valores de $\mathrm{IC}_{50}(\mu \mathrm{M})$ com intervalo de confiança de 95\% das chalconas 2, 6, 3, 4 e 1 nas linhagens AGP01 e MRC5.

\begin{tabular}{cccccccccccc}
\hline Tempo & \multicolumn{2}{c}{$\mathbf{2}$} & \multicolumn{2}{c}{ 6 } & \multicolumn{3}{c}{$\mathbf{3}$} & \multicolumn{2}{c}{$\mathbf{4}$} & \multicolumn{1}{c}{ 1 } \\
\hline & AGP01 & MRC5 & AGP01 & MRC5 & AGP01 & MRC5 & AGP01 & MRC5 & AGP01 & MRC5 \\
$\mathbf{2 4 h}$ & 19,44 & 7,39 & 5,77 & 5,17 & 7,64 & 7,46 & 9,73 & 21,05 & 9,5 & 9,88 \\
$\mathbf{4 8 h}$ & 5,16 & 4,66 & 3,11 & 3,33 & 3,86 & 4,38 & 5,1 & 4,75 & 4,11 & 2,85 \\
$\mathbf{7 2 h}$ & 4,86 & 4,11 & 2,44 & 3,07 & $\mathbf{2 , 0 8}$ & 3,57 & 4,99 & 3,3 & 4,41 & 2,75 \\
\hline
\end{tabular}

Fonte: Autores.

Todas as chalconas reduziram a viabilidade celular de forma tempo-dependente em ambas as linhagens. Na linhagem de câncer gástrico (AGP01) a chalcona com maior potencial citotóxico foi a $\mathbf{3}$, tendo uma $\mathrm{IC}_{50}$ de $2,08 \mu \mathrm{M}$ no tempo de 72 h, apresentando também os menores valores nos outros tempos de tratamento. Apesar das chalconas apresentarem $\mathrm{IC}_{50}$ baixo para a linhagem tumoral, elas também foram citotóxicas para a linhagem normal de pulmão. Entretanto, estudos com outros tipos de células normais podem apresentar resultados melhores, porque, apesar de ter diminuído a viabilidade das linhagens MRC5, pode ter um resultado diferente em linhagem de células gástricas normais, não causando a redução da viabilidade.

A partir da razão entre o IC $_{50}$ MRC5 e o IC 50 AGP01 calculou-se o índice de seletividade (IS) de cada composto, o que representa a relação entre a dose ativa e a dose tóxica. A literatura considera um bom índice de seletividade valores acima de 3,0 (Bézivin et al., 2003). Dentre as chalconas, a que apresentou o melhor efeito foi a PEB em 24h, com um IS = 2,17.

Modzelewska et al. (2006), relataram o potencial efeito inibitório de uma série de bis-chalconas no crescimento da linhagem celular HCT116 (câncer de cólon), cujos resultados evidenciaram a inibição do crescimento celular de HCT116 em comparação com as células normais, com um $\mathrm{IC}_{50}$ de $2,8 \mu \mathrm{M}$, resultado bastante semelhante ao encontrando neste trabalho.

Estudos reportados por Echeverria et al. (2009), demonstraram que a 2'-hidróxi-chalconas possuem atividades antineoplásicas e os efeitos antitumorais foram testados em linhagem celular HepG2 (câncer de fígado), com efeito indutor de apoptose e inibição da proliferação celular, entretanto não foram feitos testes em células normais.

Syam et al. (2012) sintetizaram diversas chalconas e avaliaram suas toxicidades in vitro frente a célula MCF-7 (adenocarcinoma de mama) com $\mathrm{IC}_{50}$ de $6.875 \pm 0.219 \mu \mathrm{g} / \mathrm{mL}, 7.992 \pm 0.81 \mu \mathrm{g} / \mathrm{mL}, 6.873 \pm 1.2 \mu \mathrm{g} / \mathrm{mL}$ e $5.251 \pm 0.67 \mu \mathrm{g} / \mathrm{mL}$, respectivamente da chalcona, 2-cloro-chalcona, 2-hidróxi-3-metil-chalcona, 3-cloro-chalcona, onde quatro chalconas mostraram indução de apoptose em células MCF-7.

Anwar et al. (2018) sintetizaram derivados de chalconas, sendo avaliadas in vitro contra a linhagem cancerígena de cólon (WiDr) e de mama (T47D), a 4'-hidróxi-4-cloro-chalcona apresentou $\mathrm{IC}_{50}$ de 20,42 $\mu \mathrm{g} / \mathrm{mL}$ contra a linha celular WiDr e $\mathrm{IC}_{50}$ de $57,70 \mu \mathrm{g} / \mathrm{mL}$ para a célula T47D. Porém a 4'-hidróxi-3,4-dimetóxi-chalcona foi a mais seletiva com IS de 60,22. Apesar de um ótimo índice de seletividade contra a linhagem T47D, ainda apresenta uma concentração alta com IC ${ }_{50}$ de 44,67, contudo evidencia o potencial das chalconas para um efeito anticâncer. Relataram o efeito de substituintes do anel aromático sobre seu potencial inibitório, observaram que os compostos contendo grupos retiradores de elétrons, (como nitro, flúor, cloro e bromo) mostraram maior potencial inibitório do que aqueles contendo grupos doadores de elétrons $\left(-\mathrm{OH},-\mathrm{OCH}_{3}\right)$.

A atividade das chalconas não depende apenas de sua estrutura, podem ter múltiplos alvos e diferentes mecanismos de ação, dependendo da especificidade do tumor. A seletividade das chalconas foi observada por vários trabalhos de pesquisa em diferentes linhagens de células cancerosas (Orlikova et al., 2011; Kotha et al., 2017; Sharma et al., 2016). Algumas chalconas tem grande potencial para gerar novas drogas anticancerígenas que podem agir causando danos ao DNA, inibindo a tubulina e 
fibras do fuso, indução da morte celular, inibindo a produção de vasos sanguíneos dentre outros (Das \& Manna, 2016). Principalmente aquelas em que há presença de substituinte hidroxila $(-\mathrm{OH})$ e metoxila $\left(-\mathrm{OCH}_{3}\right)$, pois a alteração de ambos os anéis influenciou na atividade dos compostos (Devi, Aswini, Kothai, apud Lakshmi, 2018).

\section{Conclusão}

As substâncias foram sintetizadas a partir da reação de Claisen-Schimidt, demostrando ser uma boa via sintética para a produção de chalconas e derivados. O teste antiproliferativo apresentou resultado significante. Para trabalhos futuros, pretende-se testar as substâncias mais ativas em outras linhagens de células.

\section{Conflito de Interesses}

Os autores declaram não haver conflito de interesses.

\section{Agradecimentos}

Os autores agradecem a todos os colaboradores dos laboratórios de Farmacologia, o núcleo de pesquisas em oncologia da UFPA e a CAPES pelo apoio a pesquisa.

\section{Referências}

Ahmad, M. R., Sastry, V. G., Bano, N. \& Anwar, S. (2016). Synthesis of novel chalcone derivatives by conventional and microwave irradiation methods and their pharmacological activities. Arab J Chem, 9, 931-935. http://dx.doi.org/10.1016/j.arabjc.2011.09.002

Alard, E., Butnariu, A-B., Grillo, M., Kirkham, C., Zinovkin, D. A., Newnham, L., Macciochi, J. \& Pranjol, M. Z. I. (2020). Advances in Anti-Cancer Immunotherapy: Car-T Cell, Checkpoint Inhibitors, Dendritic Cell Vaccines, and Oncolytic Viruses, and Emerging Cellular and Molecular Targets. Cancers, 12, 1826. DOI:10.3390/cancers12071826

Anwar, C., Prasetyo, Y. D., Matsjeh, S., Haryadi, W., Sholikhah, E. N., Nendrowati. (2018). Synthesis of Chalcone Derivatives and Their in vitro Anticancer Test against Breast (T47D) and Colon (WiDr) Cancer Cell Line. Indones. J. Chem., 18 (1), 102 - 107. DOI: 10.22146/ijc.26864

Attar, S., O’brien, Z., Alhaddad, H., Golden, M. L. \& Calderón-Urrea, A. (2011). Ferrocenyl chalcones versus organic chalcones: A comparative study of their nematocidal activity. Bioorg Med Chem, 19, 2055-2073. DOI: 10.1016/j.bmc.2011.01.048.

Bitencourt, H. R.; de Albuquerque, C. A. B.; Souza Filho, A. P. S.; dos Anjos, M. L.; Maciel, C. J. A.; Pina, J. R. S.; Pinheiro, J. C.; de Carvalho, L. L. P. P.; Marinho, A. M. R.; de Almeida, O. (2020a). Análise dos Produtos de Reação da Condensação entre 2-Hidróxiacetofenona e p-Anisaldeído em Meio Básico. In: A química nas áreas natural, tecnológica e sustentável. Vol. 3. p. 26-34. Organizadora Érica de Melo Azevedo. - Ponta Grossa, PR: Atena Editora. DOI 10.22533/at.ed.8422017093.

Bitencourt, H. R.; Marinho, A. M. R.; Souza Filho, A. P. S.; Pinheiro, J. C.; Tavares, M. G. C.; de Almeida, O; Farias, R. A. F. (2020b). Síntese de chalconas. In: Processos Químicos e Biotecnológicos. Vol. 6. p. 57-66. Organizador Darly Fernando Andrade. Belo Horizonte-MG. Editora Poisson. DOI 10.36229/97865-5866-009-5.

Bézivin, C.; Tomasi, S.; Lohézic-Le, D.; Boustie, F. J. (2003). Cytotoxic activity of some lichen extracts on murine and human cancer cell lines. Phytomedicine, 10, 499-503. Doi:10.1078/094471103322331458.

Bray, F., Ferlay, J., Soerjomataram, I., Siegel, R. L., Torre, L. A. \& Jemal, A. (2018). Global Cancer Statistics 2018: GLOBOCAN Estimates of Incidence and Mortality Worldwide for 36 Cancers in 185 Countries. CA CANCER J CLIN, 68, 394-424. DOI: 10.3322/caac.21492. Available online at cacancerjournal.com.

Das, M. \& Manna K. (2016). Chalcone Scaffold in anticancer armamentarium: a molecular insight. J. Toxicol, 2016, 1-4. http://dx.doi.org/10.1155/2016/7651047

De Mello, T. F. P.; Bitencourt, H. R.; Pedroso, R. B.; Aristides, S. M. A.; Lonardoni, M. V. C.; Silveira, T. G. V. (2014). Leishmanicidal activity of synthetic chalcones in Leishmania (Viannia) braziliensis. Exp Parasitol. 136, 27-34. Doi: 10.1016/j.exppara.2013.11.003.

Devi, L. D., Aswini, R., Kothai, S. Synthesis And Characterisation Of Chalcone Based Copolyesters And Their Anticancer Activity. (2018). IJPSR, 9(4), 1589-1593. DOI: 10.13040/IJPSR.0975-8232.9(4).1589-93

Echeverria, C., Santibañez, J. F., Donoso-Tauda, O., Escobar, C. A., Ramirez-Tagle, R. (2009). Structural Antitumoral Activity Relationships of Synthetic Chalcones. Int. J. Mol. Sci., 10, 221-231. DOI:10.3390/ijms10010221

Ferlay, J., Colombet, M., Soerjomataram, I., Mathers, C., Parkin, D.M., Piñeros, M., Znaor, A. \& Bray, F. (2019). Estimating the global cancer incidence and mortality in 2018: GLOBOCAN sources and methods. Int. J. Cancer, 144, 1941-1953. DOI: 10.1002/ijc.31937 
Fiorica, F., Trovò, M., Ottaiano, A., Nasti, G., Carandina, I., Marzola, M., Paoli, P. \& Berretta, M. (2018). Can the addition of radiotherapy postoperatively increase clinical outcome of patients with gastric cancer? A systematic review of the literature and meta-analysis. Oncotarget, 9 (12), 10734-10744. DOI: 10.18632 oncotarget. 23754

Fu, Y., Liu, D., Zeng, H., Ren, X., Song, B., Hu, B. (2020). New chalcone derivatives: synthesis, antiviral activity and mechanism of action. RSC Adv. 10, 24483-24490. DOI: 10.1039/d0ra03684f

Ismail, N. L., Ming-Tatt L, Lajis, N. L., Akhtar, M. N., Akira, A., Perimal, E. K., Israf, D. A. \& Sulaiman, M. R. (2016). Antinociceptive Effect of 3-(2,3Dimethoxyphenyl)-1- (5-methylfuran-2-yl) prop-2-en-1-one in Mice Models of Induced Nociception. Molecules, 21, 1077. DOI:10.3390/molecules21081077

Isoda, H., Motojima, H., Onaga, S., Samet, I., Villareal, M. O \& Han, J. (2014). Analysis of the erythroid differentiation effect of flavonoid apigenin on K562 human chronic leukemia cells. Chem Biol Interact, 220, 269-277. http://dx.doi.org/10.1016/j.cbi.2014.07.006

Kotha, R. R., Kulkarni, R. G., Garige, A. K., Nerella, S. G. \& Garlapati, A. (2017). Synthesis and Cytotoxic Activity of New Chalcones and their Flavonol Derivatives. Med Chem, 7 (11), 353-360. DOI: 10.4172/2161-0444.1000480

Marrelli, D., Polom, K., Manzoni, G., Morgagni, P., Baiocchi, G. L. \& Roviello, F. (2015). Multimodal treatment in gastric cancer. WJG, $21(26)$, 7954-7969. DOI:10.3748/wjg.v21.i26.7954

Marquina, S., Maldonado-Santiago, M., Sanchez-Carranza, J. N., Antunez-Mojica, M., Gonzalez-Mayal, Razo-Hernandez, R. S. \& Alvarez L. (2019). Design, synthesis and QSAR study of 2'-hydroxy-4'-alkoxy chalcone derivatives that exert cytotoxic activity by the mitochondrial apoptotic pathway. Bioorg Med Chem, 27, 43-54. https://doi.org/10.1016/j.bmc.2018.10.045

Modzelewska, A., Pettit, C., Achatanta, G., Davidson, N. E., Huang, P., Khan, S. R. (2006). Anticancer activities of novel chalcone and bis-chalcone derivatives. Bioorganic \& Medicinal Chemistry, 14, 3491-3495. DOI: 10.1016/j.bmc.2006.01.003

Mosmann, T. (1983). Rapid colorimetry assay for cellular growth and survival: application to proliferation and cytotoxicity assays. J Immunol Methods, 65, $55-63$.

Orditura, M., Galizia, G., Sforza, V., Gambardella, V., Fabozzi, A., Laterza, M. M, Andreozzi, F., Ventriglia, J., Savastano, B., Mabilia, A., Lieto, E., Ciardiello, F., De Vita, F. (2014). Treatment of gastric câncer. WJG, 20(7), 1635-1649. doi:10.3748/wjg.v20.i7.1635

Orlikova, B., Tasdemir, D., Golais, F., Dicato, M. \& Diederich, M. (2011). Dietary chalcones with chemopreventive and chemotherapeutic potential. Genes Nutr, 6, 125-147. DOI 10.1007/s12263-011-0210-5

Plummer, M., De Martel, C., Vignat, J., Ferlay, J., Bray, F. \& Franceschi, S. Global burden of cancers attributable to infections in 2012. a synthetic analysis. (2016). The Lancet Glob Health, 4, 609-616. http://dx.doi.org/10.1016/S2214-109X(16)30143-7

Sharma, R., Kumar, R., Kodwani, R., Kapoor, S., Khare, A., Bansal, R., Khurana, S., Singh, S., Thomas, J., Roy, B., Phartyal, R., Saluja, S., Kumar, S. (2016). A Review on Mechanisms of Anti Tumor Activity of Chalcones. Anti-Cancer Agents Med Chem, 16(2), 200-211. DOI: $10.2174 / 1871520615666150518093144$

Sandhar, H. K., Kumar, B., Prasher, S., Tiwari, P., Salhan, M. \& Sharma, P. (2011). A Review of Phytochemistry and Pharmacology of Flavonoids. J Pharm Sci, 1, 25-41.

Santos, M. B., Anselmo, D. B., Oliveira, J. G., Jardim-Perassi, B. V., Monteiro, D. A., Silva, G., Gomes, E., Fachin, A. L., Marins, M., Zuccari, D. A. P. C. \& Regasini, L. O. (2019). Antiproliferative activity and p53 upregulation effects of chalcones on human breast cancer cells. J Enzyme Inhib Med Chem, 34, 1093-1099. DOI: 10.1080/14756366.2019.1615485

Suwito, H., Jumina, Mustofa, Pudjiastuti, P., Fanani, M. Z., Kimata-Ariga, Y., Katahira, R., Kawakami, T., Fujiwara, T., Hase, T., Sirat, H. M. And Puspaningsih, N. N. T. (2014). Design and Synthesis of Chalcone Derivatives as Inhibitors of the Ferredoxin - Ferredoxin-NADP+ Reductase Interaction of Plasmodium falciparum: Pursuing New Antimalarial Agents. Molecules, 19, 21473-21488. DOI:10.3390/molecules191221473

Syam, S., Abdelwahab, S. I., Al-Mamary, M. A. And Mohan, S. (2012). Synthesis of Chalcones with Anticancer Activities. Molecules, 17, 6179-6195. DOI: $10.3390 /$ molecules 17066179

Tomar, V., Bhatacharjee, G., Kamaluddin, Rajakumar, S., Srivastava, K. \& Puri, S. K. (2010). Synthesis of new chalcone derivatives containing acridinyl moiety with potential antimalarial activity. Eur. j. med. Chem, 45, 745-751. DOI:10.1016/j.ejmech.2009.11.022

Ventura, T. L. B., Calixto, S. D., Abrahim-Vieira, B. A., Souza, A. M. T., Mello, M. V. P., Rodrigues, C. R., Miranda, L. S. M., Souza, R. O. M. A., Leal, I. C. R., Lasunskaia, E. B. \& Muzitano, M. F. (2015). Antimycobacterial and Anti-Inflammatory Activities of Substituted Chalcones Focusing on an AntiTuberculosis Dual Treatment Approach. Molecules, 20, 8072-8093. DOI:10.3390/molecules20058072

Wang, T-Y, Li, Q. \& Bi, K-S. (2018). Bioactive flavonoids in medicinal plants: Structure, activity and biological fate. Asian J Pharm, 13, 12-23. https://doi.org/10.1016/j.ajps.2017.08.004

WHO. 2018. International Agency for Research on Cancer. World Cancer Report: 2020. https://www.who.int/health-topics/cancer\#tab=tab_1.

Wu, J., Li, J., Cai, Y., Pan, Y., Ye, F., Zhang, Y., Zhao, Y., Yang, S., Li, X. \& Liang, G. (2011). Evaluation and discovery of novel synthetic chalcone derivatives as anti-inflammatory agents. J. Med. Chem, 5, 8110-8123. http://dx.doi.org/10.1021/jm200946h

Yadav, P., Lal, K., Kumar, A., Guru, S. K., Jaglan, S. \& Bhushan, S. (2017). Green synthesis and anticancer potential of chalcone linked-1,2,3-triazoles. Eur. j. med. Chem, 126, 944-953. http://dx.doi.org/10.1016/j.ejmech.2016.11.030

Zhang, H., Jin, H., Ji, L-Z.., Tao, K., Liu, W., Zhao, H-Y. \& Hou, T-P. (2011). Design, Synthesis, and Bioactivities Screening of a Diaryl Ketone-Inspired Pesticide Molecular Library as Derived from Natural Products. Chem Biol Drug Des, 78, 94-100. DOI: 10.1111/j.1747-0285.2011.01082.x. 